\title{
Estimation of the Longitudinal Impedance of the ATF Damping Ring
}

\author{
M. Takao, T. Higo, K. Kanazawa, H. Nakayama, J. Urakawa and K. Yokoya \\ National Laboratory for High Energy Physics (KEK), Tsukuba, Ibaraki 305, Japan \\ K. L. F. Bane \\ Stanford Linear Accelerator Center, Stanford, California 94309, USA
}

\begin{abstract}
In order to estimate the possibility of bunch lengthening in the ATF damping ring, the longitudinal broadband impedance of the ring was evaluated. The inductance was so small, that the bunch lengthening should not be observed in the ATF damping ring.
\end{abstract}

\section{INTRODUCTION}

As a check point to the Japan Linear Collider (JLC) [1], it is planned to construct Accelerator Test Facility (ATF). The damping ring, one of the main parts of ATF, has the almost same as that of JLC design. The vacuum chamber of the damping ring has many small discontinuities - vacuum pumping holes, bellows, monitor electrodes and so on - which are normally inductive. The induced voltage by a bunch passing through an inductive object has the slope of the opposite sign to RF field. Then, operating a damping ring at high current can result in significant bunch lengthening [2] if the longitudinal broadband impedance of the ring is larger than a critical value. Therefore we estimate the longitudinal impedances of the various components in the damping ring by $T B C I$ [3] and $T 3$ [4] in MAFIA codes.

The parameters of the ATF damping ring, which are necessary in estimating the impedances of the ring, are listed in Table 1.

Table 1. Parameters of ATF damping ring

\begin{tabular}{|c|c|}
\hline Beam Energy & $1.51 \mathrm{GeV}$ \\
\hline Circumference & $147.8 \mathrm{~m}$ \\
\hline Longitudinal Impedance Threshold & $0.78 \Omega$ \\
\hline Momentum Compaction & 0.0035 \\
\hline Accelerating Frequency & $1.128 \mathrm{GHz}$ \\
\hline Current & $\sim 200 \mathrm{~mA}$ \\
\hline Bunch Length & $4.72 \mathrm{~mm}$ \\
\hline Energy Spread & $0.08 \%$ \\
\hline
\end{tabular}

\section{WAKE FIELD AND IMPEDANCE}

Before calculating impedances of the ATF damping ring, we review the definitions of the longitudinal wake field and the impedance [5]. Denoting the impulse wake function, i.e. the wake function for a single particle, as $W_{\|}^{(0)}(s)$, we can express the longitudinal wake function
$W_{\|}(s)$ for the bunch with an arbitrary longitudinal charge distribution $\lambda(s)$ as

$$
W_{\|}(s)=\frac{1}{Q} \int_{-\infty}^{\infty} d s^{\prime} \lambda\left(s-s^{\prime}\right) W_{\|}^{(0)}\left(s^{\prime}\right)
$$

The longitudinal impedance $Z_{\|}(\omega)$ is the Fourier transform of the longitudinal wake function $W_{\|}^{(0)}(s)$;

$$
Z_{\|}(\omega)=\frac{1}{c} \int_{-\infty}^{\infty} d s e^{i \omega s / c} W_{\|}^{(0)}(s)
$$

The impedance can be decomposed into the real and imaginary parts as

$$
Z_{\|}(\omega)=\mathcal{R}_{\|}(\omega)+i \mathcal{X}_{\|}(\omega)
$$

The former is called the resistance and the latter the reactance.

The loss factor is defined by the self energy loss in the bunch and can be expressed in terms of the integral over the resistance;

$$
k_{\|}=\frac{c^{2}}{\pi Q^{2}} \int_{0}^{\infty} d \omega \tilde{\lambda}^{2}(\omega) \mathcal{R}_{\|}(\omega)
$$

where $\tilde{\lambda}(\omega)$ is the Fourier transform of $\lambda(s)$. In this note, we only consider the gaussian bunch $(\lambda(s)=$ $\frac{Q}{\sqrt{2 \pi} \sigma_{z}} e^{-s^{2} / 2 \sigma_{z}^{2}}$, where $\sigma_{z}$ is the r.m.s. bunch length). In the following calculation, the bunch length is assumed to be $5 \mathrm{~mm}$ for the sake of adjusting mesh size.

\section{CALCULATION OF THE IMPEDANCE OF ATF DAMPING RING}

\section{A. Vacuum Pump Holes in Normal Section}

In the normal section the beam pipe has the radius of $13.5 \mathrm{~mm}$, and the thickness of the pipe is $1 \mathrm{~mm}$. To keep the vacuum necessary for the ATF darnping ring, we need 15 vacuum pumping holes per $1 \mathrm{~cm}$ length of the pipe if the diameter of the hole is $1 \mathrm{~mm}$. Using the 3 dimensional code T3 in MAFIA [4], we can estimate the contribution of a single hole to the longitudinal wake field. Figure 1 shows the wake field of the hole with radius $2 \mathrm{~mm}$. 


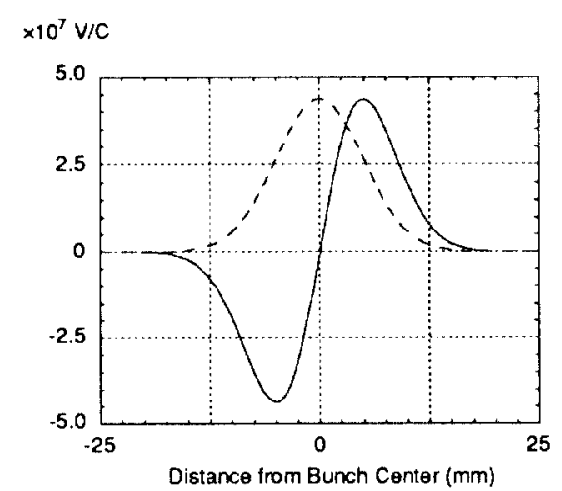

Figure 1. Longitudinal wake field of a hole with a radius of $2 \mathrm{~mm}$ excited by a gaussian bunch of $\sigma_{z}=$ $5 \mathrm{~mm}$ in the ATF damping ring.

In this case the impulse wake potential was found approximately proportional to the derivative of $\delta$-function

$$
W_{\|}^{(0)}(s)=c^{2} L \delta^{\prime}(s)
$$

where $L$ is a constant identified with the inductance. Therefore, from Eq.(2) we find that the effective impedance is inductive in character;

$$
Z_{\|}(\omega)=-i \omega L .
$$

On the other hand, the total wake potential (1) is now written as

$$
W_{\|}(s)=\frac{c^{2} L}{\sqrt{2 \pi} \sigma_{z}^{3}} s e^{-s^{2} / 2 \sigma_{z}^{2}}
$$

The effective inductance is then determined by the absolute maximum $W_{\|}^{(\max )}$ of the wake field $W_{\|}(s)$ as

$$
L=\sqrt{2 \pi} e^{1 / 2} \sigma_{z}^{2} W_{\|}^{(\max )} / c^{2} .
$$

As a result, we can calculate $\left|Z_{||}(\omega) / n\right|$ ( $n$ is the ratio of $\omega$ to the revolution frequency $\omega_{0}$ ) through the effective inductance. The impedances of holes with various radii were calculated and shown in Fig.2. The figure shows that the impedance obeys the third power law of the radius.

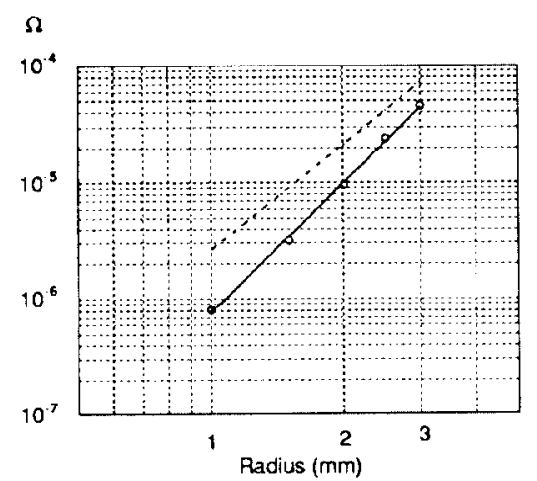

Figure 2. Dependence of the inductance of a small hole on the radius. Solid line indicates the calculated impedance. Dashed line corresponds to the approximate formula of Eq.(9).
The wake field of two holes separated by the same distance as thcir diameter was twice as that of a single hole, showing no interference between them.

Let us now interpret physically the inductance of a small hole by the following simple argument. Consider a small cylindrical cavity on a beam pipe. If the magnetic field is unperturbed at the cavity, the inductance is given by $[5,6]$

$$
L=\frac{Z_{0}}{2 \pi c} \frac{g \Delta}{a},
$$

where $a$ is the radius of the beam pipe, $g$ the gap length and $\Delta$ the depth of the cavity $(a \gg \Delta)$. Let us rewrite the above expression into the one for the small hole. Since in a cylindrical hole electromagnetic fields attenuate as $\exp \left(-\frac{y}{r / j_{01}}\right)$, where $r$ is the radius of the hole, $y$ the distance from the beam pipe surface, the depth of the gap $\Delta$ should be replaced with the attenuation length in the hole $r / j_{01}$. Taking an azimuthal filling factor of the hole into account, we can replace $g$ with $r^{2} / 2 a$ and get the expression for the impedance of a small hole as

$$
\left|Z_{\|}(\omega) / n\right|=\frac{\omega_{0} Z_{0}}{4 \pi j_{01} c} \frac{r^{3}}{a^{2}},
$$

which is plotted by the dashed line in Fig.2. The approximate formula gives a proper power law. The magnitude is larger by about factor 2 than the one computed by $T$ ? This is because the effect of the current detour from the small hole is not taken into account in the above simple argument.

\section{B. Vacuum Pump Slots in Wiggler Section}

Next we consider the impedance of the vacuum system at the wiggler part, which has the cross section depicted in Fig.3. The beam pipe is pumped through the two slots on the right hand side. To hold the cooling water guide, there is a support width of $5 \mathrm{~mm}$ in every $35 \mathrm{~mm}$. The calculation by $T 3$ shows that the longitudinal impedance is also inductive. To see the dependence of the slot on the longitudinal length, we compute the impedance of slots with various lengths. As the longitudinal length of slot is enlarged from $10 \mathrm{~mm}$ to $30 \mathrm{~mm}$, the amount of the impedance $|Z / n|$ slightly changes from $5 \times 10^{-7} \Omega$ to $6 \times 10^{-7} \Omega$.

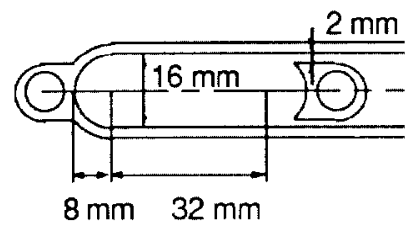

Figure 3. Cross section of the vacuum chamber at the wiggler part

\section{Monitor Electrodes}

In evaluating the impedance of the monitor electrode of button type (a radius of the outer cylinder is $2.5 \mathrm{~mm}$, and that of the button electrode $2 \mathrm{~mm}$ ), we have used a coaxial wave guide as a model of the electrode. The outer 
radius of the wave guide is fixed to $2.5 \mathrm{~mm}$. Since the clcctrode is a small discontinuity, the impedance is also inductive. By computing the impedances for the various inner radii, we found that the impedance scarcely depends on the inner radius of the coaxial wave guide.

\section{Bellows}

The bellows are shielded, so that the beam only sees two shallow transition instead of a corrugated structure. Although there are some slits on the shielding, the slits are narrow enough to give little contribution to the impedance. On the analogy of pumping hole, a single slit on the shielding contributes to the inductance by $\sim 10^{-7} \Omega$, if the width of the slit is $1 \mathrm{~mm}$ while independent on its length. The impedance of the shallow transition is still inductive, and an approximate formula for the inductance of a pair of shallow transitions [2];

$$
L=\frac{3 Z_{0}}{2 \pi c} \frac{a \Delta^{2}}{b}\left(\frac{2 \theta}{\pi}\right)^{1 / 2},
$$

is applicable. Here $a$ is a beam pipe radius, $\Delta$ the depth of shallow, $b-a+\Delta$, and $\theta(0<\theta \leq \pi / 2)$ the angle of a shallow transition. In our case, a half of the factor for transition angle $(2 \theta / \pi)^{1 / 2}$ should be replaced with 1 , since at one end the beam pipe changes at right angle. Inserting our parameter into the formula, we found that the impedance of one shielded bellows unit was $2.7 \times 10^{-4}$ $\Omega$. In order to confirm the above result, the impedance was calculated by $T B C I$. The impedance is regarded as inductive and the amount is $\left|Z_{\|} / n\right| \sim 3.5 \times 10^{-4} \Omega$ for a shallow transition.

\section{E. Other Inductive Elements}

The remainders of the estimation are the septa and the $R F$ quadrupole, each of which has a complicated structure. Using the code TY in MAFIA, we calculated the wake fields of septa and RF quadruple and found that they were small inductive elements. The inductance of the former was $6.8 \times 10^{-1} \Omega$ and that of the latter $6.4 \times 10^{-3} \Omega$.

\section{F. RF Cavities}

It is considered that the RF cavity is most important for the resistive wake. Using the code TBCI [3], we evaluated the longitudinal wake field for a possible design of the RF cavity for the ATF damping ring (see Fig.4). Since the wake function approximates the negative of the bunch shape, we can regard the effective impedance, for our bunch length, to be purely resistive in character with constant resistance $R_{\|}$. Then $\mathcal{R}_{\|}$is written in terms of the loss factor $k_{\|}\left(\sigma_{z}\right)$ as

$$
\mathcal{R}_{\|}=2 \sqrt{\pi} \frac{\sigma_{z}}{c} k_{\|}\left(\sigma_{z}\right)
$$

Taking the parameter $c / \sigma_{z}$ as characteristic frequency $\omega$ for the impedance of the gaussian bunch, the longitudinal impedance is expressed by the resistance [2];

$$
\left|Z_{\|}(\omega) / n\right| \sim \frac{\sigma_{z} \omega_{0}}{c} \mathcal{R}_{\|}
$$

For our bunch length, the loss factor $k\left(\sigma_{z}\right)$ of the RF cavity was found $1.6 \mathrm{~V} / \mathrm{pC}$, then the Iongitudinal impedance $\left|Z_{||} / n\right|$ of the single cavity was $0.02 \Omega$. The impedance contributed by RF cavities, 4 or 8 of which we need in the ATF damping ring, is $0.08 \Omega$ or $0.16 \Omega$, respectively, i.e. below the threshold value.

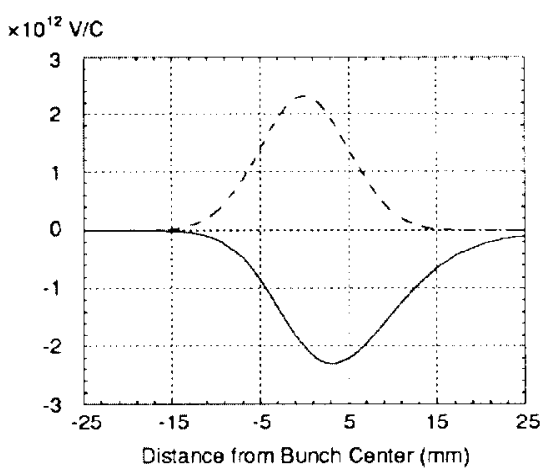

Figure 4. Longitudinal wake field of RF cavity.

\section{SUMMARY}

The results for the elements of the ATF damping ring are listed in Table 2.

Table 2. Impedances of ATF damping ring

\begin{tabular}{|c|c|c|c|}
\hline \multicolumn{2}{|c|}{ Single Element Impedance } & \multicolumn{2}{|c|}{ Total Impedance } \\
\hline Components & $\left|Z_{\|} / n\right|(\Omega)$ & Number & $\left|Z_{\|} / n\right|(\Omega)$ \\
\hline cavities & 0.02 & 4 or 8 & 0.08 or 0.16 \\
\hline $\begin{array}{c}\text { ion pump holes } \\
\text { (normal part) }\end{array}$ & $5.4 \times 10^{-8}$ & $\begin{array}{c}15 \text { per } 1 \mathrm{~cm} \\
\text { over } 100 \mathrm{~m}\end{array}$ & $8 \times 10^{-3}$ \\
\hline $\begin{array}{c}\text { ion pump slots } \\
\text { (wiggler part) }\end{array}$ & $6 \times 10^{-7}$ & $\begin{array}{c}1 \text { per } 3.5 \mathrm{~cm} \\
\text { over } 40 \mathrm{mI}\end{array}$ & $7 \times 10^{-4}$ \\
\hline monitor & $2 \times 10^{-5}$ & $4 \times 130$ & 0.01 \\
\hline bellows & $3.5 \times 10^{-4}$ & 118 & 0.04 \\
\hline others & & & 0.01 \\
\hline
\end{tabular}

In conclusion, we found that the total $\left|Z_{\|} / n\right|$ is less than $0.3 \Omega$. Therefore bunch lengthening should not be a problem in the ATF damping ring.

\section{REFERENCES}

[1] S. Takeda, Proc. First Workshop of Japan Linear Collider, Tsukuba, (1989).

[2] K.L.F. Bane, Bunch Lengthening in the SLC Damping Ring, SLAC-PUB-5177 (1990).

[3] T. Weiland, Nucl.Instrum.Methods 212 (1983) 13.

[4] R. Klatt and T. Weiland, Procecdings of Linear Accelerator Conference, SLAC, (1986) 282.

[5] K.L.F. Bane and M. Sands, Wakefields of Very Short Bunches in an Accelerating Cavity, SLAC-PUB-4441 (1987).

[6] E. Keil and B. Zotter, Particle Accelerators 3 (1972) 11. 